\title{
An Index Formula for Production Economies with Externalities.
}

\author{
Antoine Mandel ${ }^{1}$ \\ Potsdam Institute for Climate Impact Research \\ Telegrafenberg A51, 14473 Potsdam, Germany \\ Tel 493312882572 Fax 493312882640 \\ email : antoine.mandel@pik-potsdam.de \\ Paris School of Economics, University Paris 1 Panthéon-Sorbonne \\ 106-112 Boulevard de l'hôpital 75013 Paris, France
}

\begin{abstract}
We prove that the degree of the equilibrium correspondence of an economy with increasing returns and external effects is equal to $(-1)^{L-1}$ where $L$ is the dimension of the space of goods. This allows us to infer existence, finiteness and uniqueness results.
\end{abstract}

Key Words: General Equilibrium Theory, Existence of Equilibrium, Increasing Returns, Externalities, Degree Theory.

JEL Codes: C62, D51, D62

\footnotetext{
1 The author is currently affiliated to the Potsdam Institute, however this article was mainly written during the author Ph.D thesis at the Paris School of Economics under the supervision of Pr. Jean-Marc Bonnisseau, whom the author would like to thank for his guidance and numerous advices. Also the comments of an anonymous referee and of the guest editor led to substantial improvement of the paper. All remaining errors are mine.
} 


\section{Introduction}

In this paper we construct an homotopy between the equilibrium correspondence of an economy with external effects and increasing returns and the equilibrium correspondence of a standard ${ }^{2}$ economy with increasing returns. This construction allows us to show that the degree of the equilibrium correspondence with externalities is equal to $(-1)^{L-1}$ where $L$ is the dimension of the space of goods, and hence to infer existence, finiteness and uniqueness results. It also allows us to analyze the adaptation of an economy to long term environmental changes such as climate change.

As emphasized by the title of Starret's paper (Starret 1972), "Fundamental non-convexities in the theory of externalities", external effects and increasing returns are closely related phenomena, especially when the economy encompasses markets of allowances for external effects. Existence of equilibrium in such a setting has already been studied by (Bonnisseau and Médecin 2001) and (Bonnisseau 1997) for general pricing rules, while (Laffont 1978) deals with the case of profit maximizing producers. Index formula have been established in exchange economies with externalities by (Bonnisseau 2003) and (Del Mercato 2006) but, to our knowledge, these results have never been extended to production economies and hence there remains an important indeterminacy on the structure of the set of equilibria of those economies.

On another hand, the standard treatment of externalities in general equilibrium theory as directed phenomena (see (Laffont 1978)) partially lacks generality to model the major issue that climate change today represents. Indeed, in this framework an agent can not have an external effect on himself. This seems to render problematic the satisfaction of the free-disposal assumption in presence of external effects and probably is not an appropriate model in the case of climate change, as the atmospheric concentration of greenhouse gases increases identically whoever may be the agent who burns fossil fuels.

In order to tackle these issues, we introduce explicitly a concept of environment which corresponds to the physical framework of the economy. This environment influences the production and consumption possibilities and may in turn be influenced by the agent's choices. When this influence is unilateral (from the environment to the economy), we can use the results of Jouini for standard economies (Jouini 1992a), in order to compute the degree of the equilibrium correspondence for a given environment. From the point of view of interpretation, this given initial environment is seen as the situation that prevails before the feedbacks of greenhouse gases emissions on the climate become economically meaningful (i.e the initial environment under consideration is the current,

$\overline{2}$ In the remaining of the paper the standard case is this of an economy with increasing returns but without external effects. 
or recent past, one).

The influence of the economic activity on the environment is progressively introduced via an homotopy. The index formula can then be extended to the framework with external effects provided the economy satisfies a survival assumption for each environment it may face along the way. The main result of the paper, stated as Theorem 2, hence gives conditions which ensure the economy can adapt to major environmental changes. Moreover it enables us, provided a suitable notion of regularity is introduced, to limit the indeterminacy on the equilibria that can be reached.

The general organization of the paper is as follows. In section 2, we present the general characteristics of the model, introduce the concept of environment and define the notions of equilibrium for a fixed environment and of equilibrium with external effects. In section 3, after having presented the survival assumptions, we construct the equilibrium correspondences with and without external effects as well as the homotopy between these correspondences. In section 4 after having applied Jouini's result to compute the degree with fixed environment in Theorem 1, we use the homotopy invariance property of the degree to show, in Theorem 2, that the degree of the equilibrium correspondence with external effects is equal to $(-1)^{L-1}$, where $L$ is the number of goods in the economy. We then derive as corrolaries existence results. In section 5 we state additional regularity and transversality conditions in order to ensure that the set of equilibria has a manifold structure, so that almost every economy is regular. This allows us to conclude by stating finiteness and uniqueness results.

\section{The Model}

\subsection{Agents' characteristics}

We consider an economy with a finite number, $m$, of consumers indexed by $i=1 \cdots m$ and a finite number, $n$, of producers indexed by $j=1 \cdots n$. Those agents consume, produce and exchange a finite number $L$ of commodities according to prices $p$ in the simplex of $\mathbb{R}^{L}$. The economy is lying within an environment whose state is described by a vector of $E$ real parameters $e \in \mathbb{R}^{E}$, and there are reciprocal interactions between the economic activity and the environment. ${ }^{3}$

3 Notations: $S=\left\{p \in \mathbb{R}_{+}^{L} \mid \sum_{\ell=1}^{L} p_{\ell}=1\right\}$ is the simplex of $\mathbb{R}^{L}, S_{++}$denotes its interior and $\mathcal{H}$ the affine space it spans; 1 is the vector $\left(\frac{1}{L}, \cdots, \frac{1}{L}\right) \in \mathbb{R}^{L}$. Given a set $Z, \operatorname{int}(Z)$ denotes its interior, $\operatorname{clc}(Z)$ its closed and convex closure, $\mathcal{A}(Z)$ its 
The production possibilities are described by a correspondence $Y_{j}: \mathbb{R}^{E} \rightarrow \mathbb{R}^{L}$ which associate to an environment a set of technically feasible production plans. This correspondence is assumed to satisfy the following conditions :

\section{Assumption (P)}

(1) For all $j, Y_{j}$ is a lower semi-continuous correspondence with closed graph;

(2) For all $j$, for all $e \in \mathbb{R}^{E}, Y_{j}(e)-\mathbb{R}_{+}^{L} \subset Y_{j}(e)$;

(3) $\mathcal{A}\left(\prod_{j=1}^{n} \bigcup_{e \in \mathbb{R}^{E}} Y_{j}(e)\right) \cap\left\{\left(y_{j}\right) \in\left(\mathbb{R}^{L}\right)^{n} \mid \sum_{j=1}^{n} y_{j} \geq 0\right\}=\{0\}$.

$\mathrm{P}(1)$ is a technical regularity assumption on the production correspondences, $\mathrm{P}(2)$ states that firms can freely-dispose of commodities, $\mathrm{P}(3)$ will ensure the boundedness of the set of allocations which are attainable for some environment. On the other hand, as we want to encompass the possibility of increasing returns, no convexity assumption is made. We therefore do not set the producers' behavior as profit maximization but use the more general notion of pricing rule. The pricing behavior of agent $j$ is influenced by the environment and hence described by a correspondence $\phi_{j}$ defined on the graph of the correspondence $\partial Y_{j}$,

$$
\text { Graph } \partial Y_{j}:=\left\{\left(e, y_{j}\right) \in \mathbb{R}^{E} \times \mathbb{R}^{L} \mid y_{j} \in \partial Y_{j}(e)\right\},
$$

and with values in the $L$-dimensional simplex $S$.

This pricing rule defines the producer's behavior as follows : the price $p$ is acceptable for firm $j$ given an environment $e \in \mathbb{R}^{E}$ and a production plan $y_{j} \in \partial Y_{j}(e)$ if $p \in \phi_{j}\left(e, y_{j}\right)$. We shall consider pricing rules which satisfy the following standard requirements:

\section{Assumption (PR)}

(1) For all $j, \phi_{j}$ is an upper semi-continuous, convex and compact valued correspondence from Graph $\partial Y_{j}$ to $S$.

(2) For all $j$, one of the following holds:

- $\phi_{j}$ has bounded losses (i.e there exists $\alpha_{j} \in \mathbb{R}$ such that for all $\left(e, y_{j}\right) \in$ Graph $\partial Y_{j}$ and for all $p \in \phi_{j}\left(e, y_{j}\right)$ one has $\left.p \cdot y_{j} \geq \alpha_{j}\right)$

- $\phi_{j}$ is the marginal pricing rule (i.e for all $\left(e, y_{j}\right) \in \operatorname{Graph} \partial Y_{j}, \phi_{j}\left(e, y_{j}\right)=$ $\left.N_{Y_{j}(e)}\left(y_{j}\right) \cap S.\right)$

Note that profit maximizing behavior is encompassed in this setting when the $Y_{j}$ have convex values and the elements of $\phi_{j}\left(e, y_{j}\right)$ are normal vectors to $Y_{j}(e)$ at $y_{j}$.

asymptotic cone and $N_{Z}(z)$ its Clarke's normal cone in $z$. 
The consumers' wealth comes from an initial endowment $\omega_{i} \in \mathbb{R}_{+}^{L}$ and from shares of firm's profits or losses they receive according to the revenue functions $r_{i}\left(p,\left(y_{j}\right)\right)$ defined on $S \times\left(\mathbb{R}^{L}\right)^{n}$. This wealth does not depend directly on the environment. However consumers' well-being depends on the state of the environment $e \in \mathbb{R}^{E}$ as well as on their consumption of a bundle of commodities $x_{i}$ in $\mathbb{R}_{+}^{L}$. It is measured by an utility function $u_{i}$ defined on $\mathbb{R}^{E} \times \mathbb{R}_{+}^{L}$. Consumers' characteristics are assumed to satisfy the following conditions :

\section{Assumption (C) For all $i$ :}

(1) $u_{i}$ is continuous;

(2) For all $e \in \mathbb{R}^{E}, u_{i}(e, \cdot)$ is quasi-concave;

(3) For all $e \in \mathbb{R}^{E}, u_{i}(e, \cdot)$ is strictly monotone with regards to commodities: $\forall x_{i} \in \mathbb{R}_{+}^{L}, \forall \xi \in \mathbb{R}_{+}^{L} /\{0\}, u_{i}\left(e, x_{i}\right)<u_{i}\left(e, x_{i}+\xi\right)$

(4) $r_{i}$ is continuous and for all $\left(p,\left(y_{j}\right)\right) \in S \times\left(\mathbb{R}^{L}\right)^{n}$, one has $\sum_{i=1}^{m} r_{i}\left(p,\left(y_{j}\right)\right)=p \cdot \sum_{j=1}^{n} y_{j}$.

Under assumption $\mathrm{C}$, we can sum up the behavior of the consumers by a demand correspondence:

Definition 1 The demand of agent $i$,

$$
D_{i}: \mathbb{R}^{E} \times S_{++} \times \mathbb{R}_{++} \rightarrow \mathbb{R}_{+}^{L}
$$

is the correspondence which associates to an environment $e \in \mathbb{R}^{E}$, a price $p \in S_{++}$, and a wealth $w>0$, the set of elements $\bar{x}_{i} \in \mathbb{R}_{+}^{L}$ which maximize $u_{i}(e, \cdot)$ in the budget set $B(p, w)=\left\{x_{i} \in \mathbb{R}_{+}^{L} \mid p \cdot x_{i} \leq w\right\}$.

This demand correspondence satisfies the following standard conditions :

Lemma 1 Under assumption $C$

(1) $D_{i}$ is an upper semi-continuous correspondence with non-empty convex compact values.

(2) For every $(e, p, w) \in R^{E} \times S_{++} \times \mathbb{R}_{++}$, and every $x_{i} \in D_{i}(e, p, w)$, one has $p \cdot x_{i}=w$

(3) For every $e \in \mathbb{R}^{E}$, if $\left(p_{n}, w_{n}\right)$ is a sequence in $S_{++} \times \mathbb{R}_{++}^{L}$ converging to $(p, w)$ such that $w>0$ and $p \notin S_{++}$then $\mathbf{1} \cdot D_{i}\left(e, p_{n}, w_{n}\right) \rightarrow+\infty$ 


\subsection{Definition of equilibria}

When an environment $e_{0} \in \mathbb{R}^{E}$ is given, we can define an economic equilibrium of $\mathcal{E}(\omega)^{4}$ as:

Definition 2 (Equilibrium for a fixed environment) An equilibrium of $\mathcal{E}(\omega)$ for the environment $e_{0}$ is an element $\left(\bar{p},\left(\bar{x}_{i}\right),\left(\bar{y}_{j}\right)\right) \in S_{++} \times\left(\mathbb{R}_{+}^{L}\right)^{m} \times\left(\mathbb{R}^{L}\right)^{n}$ such that:

(1) For all $i, \bar{x}_{i} \in D_{i}\left(e_{0}, \bar{p}, \bar{p} \cdot \omega_{i}+r_{i}\left(\bar{p},\left(\bar{y}_{j}\right)\right)\right.$;

(2) For all $j, \bar{y}_{j} \in \partial Y_{j}\left(e_{0}\right)$ and $\bar{p} \in \phi_{j}\left(e_{0}, \bar{y}_{j}\right)$;

(3) $\sum_{i=1}^{m} \bar{x}_{i}=\sum_{j=1}^{n} \bar{y}_{j}+\sum_{i=1}^{m} \omega_{i}$.

Such an equilibrium corresponds to a situation where there is no feedback of the economic activity on the environment. In the framework of long term environmental changes, such as climate change, it should be considered as the situation which prevails before the environmental consequences of economic activity have entered the economic timeframe.

From a more technical point of view, this definition coincide with this of an equilibrium without external effects, see e.g (Bonnisseau and Cornet 1988), which would be parametrized by the exogenously given environmental parameter $e_{0}$.

We then consider the effects of the economic activity on the environment become progressively observable and economically meaningful. At some point, they become relevant for the determination of the agents' economic choices.

In order to represent the emergence of these feedbacks, we introduce a continuous environmental function

$$
\xi:\left(\mathbb{R}^{L}\right)^{m+n} \rightarrow \mathbb{R}^{E}
$$

which continuously associate ${ }^{5}$ to a vector of consumption plans $\left(x_{i}\right) \in\left(\mathbb{R}_{+}^{L}\right)^{m}$ and production plans $\left(y_{j}\right) \in \prod_{j=1}^{n} \bigcup_{e \in \mathbb{R}^{E}} Y_{j}(e)$, the resulting state of the environment $\xi\left(\left(x_{i}\right),\left(y_{j}\right)\right)$.

Remark 1 The standard treatment of externalities à la (Laffont 1978) is encompassed in our framework when $\xi$ is the identity function on $\left(\mathbb{R}^{L}\right)^{m+n}$ and when the influence of the environment on an agent characteristics only

\footnotetext{
4 We denote by $\mathcal{E}(\omega)$ the economy where the consumer's initial endowments are set equal to $\omega=\left(\omega_{i}\right)_{i=1 \cdots m} \in\left(\mathbb{R}_{+}^{L}\right)^{m}$

5 The function is arbitrarily extended to $\left(\mathbb{R}^{L}\right)^{m+n}$ for sake of technical simplicity. However, we shall use an interpretation only when the $\left(x_{i}\right),\left(y_{j}\right)$ correspond to actual agents' choices.
} 
depends on the parameters corresponding to the other agents choices (see (Bonnisseau and Médecin 2001)). Hence in the standard setting, the environmental consequences of one agent choices on himself are a priori internalized. It seems to us, this a priori internalization renders problematic the satisfaction of the free-disposal assumption : an agent might not dispose of an input/output whose use causes him external effects without seeing its production possibilities change (see the example in the appendix). Moreover, this framework does not seem appropriate to analyze global environmental externalities such as climate change where the arm caused by the pollution is totally independent of its source.

We then define a notion of equilibrium with external effects where the ecoenvironmental feedbacks are taken into consideration:

Definition 3 (Equilibrium with external effects) An equilibrium with external effects of $\mathcal{E}(\omega)$ is an element $\left(\bar{p},\left(\bar{x}_{i}\right),\left(\bar{y}_{j}\right)\right) \in S_{++} \times\left(\mathbb{R}_{+}^{L}\right)^{m} \times\left(\mathbb{R}^{L}\right)^{n}$ such that:

(1) For all $i, \bar{x}_{i} \in D_{i}\left(\bar{e}, \bar{p}, \bar{p} \cdot \omega_{i}+r_{i}\left(\bar{p},\left(\bar{y}_{j}\right)\right)\right.$;

(2) For all $j, \bar{y}_{j} \in \partial Y_{j}(\bar{e})$ and $\bar{p} \in \phi_{j}\left(\bar{e}_{\bar{y}} \bar{y}_{j}\right)$;

(3) $\sum_{i=1}^{m} \bar{x}_{i}=\sum_{j=1}^{n} \bar{y}_{j}+\sum_{i=1}^{m} \omega_{i}$;

$$
\text { with } \bar{e}=\xi\left(\left(\bar{x}_{i}\right),\left(\bar{y}_{j}\right)\right)
$$

In the following, we shall take as a reference point an equilibrium with fixed environment and try to characterize if and how the economy can reach an equilibrium with external effects. More precisely, we will use the index formula for production economies given in (Jouini 1992a) in order to obtain an index formula for equilibria with fixed environment and will then determine which additional conditions ensure that a similar index formula hold for equilibria with external effects. This result will first be used to discuss how the existence of an equilibrium with external effects can be inferred from the existence of an equilibrium with fixed environment. Second, we will try to quantify the indeterminacy on the equilibrium with external effects which is reached after the environmental feedbacks have appeared. Hence we tend to provide a general equilibrium analysis of the conditions under which the economy can adapt to long term environmental changes.

\section{Characterization of Equilibria}

In this section, we set up an algebraic framework for the analysis of the equilibria of $\mathcal{E}(\omega)$ by characterizing them as zeroes of upper-semi-continuous correspondences. In order to construct this framework we shall first define a do- 
main for the correspondences which is appropriate in terms of mathematical regularity and also meaningful from the economic point of view (the latter is mainly achieved via the use of the survival assumptions introduced below). On another hand, we will need to adjust the representations of the agent's behavior at the margin of this domain and will therefore introduce some auxiliary contructions.

\subsection{Definition of the domain}

We first present two technical remarks which will prove useful to represent the producer's behavior :

- Using the fact that the consumption sets are bounded below and assumption $P(3)$, it is standard to show that there exists a compact ball of $\mathbb{R}^{L}, K$, such that the allocations attainable for some environment,

$$
\left\{\left(\left(x_{i}\right),\left(y_{j}\right)\right) \in \prod_{i=1}^{m} \mathbb{R}_{+}^{L} \times \prod_{j=1}^{n} \bigcup_{e \in \mathbb{R}^{E}} Y_{j}(e) \mid \sum_{j=1}^{n} y_{j}+\sum_{i=1}^{m} \omega_{i}=\sum_{i=1}^{m} x_{i}\right\}
$$

lie in the interior of $K^{m+n}$. Due to the continuity of $\xi$, there then exists a compact ball $K_{E}$ of $\mathbb{R}^{E}$, such that $\xi\left(K^{m+n}\right) \subset K_{E}$.

- According to Lemma 5 in (Bonnisseau and Cornet 1988 ), assumption $P(i i)$ implies that for all $e \in \mathbb{R}^{E}$ the restriction of $\operatorname{proj}_{1^{\perp}}$ to $\partial Y_{j}(e)$ is an homeomorphism. Its inverse $\Lambda_{j}(e, \cdot)$ is obtained by associating to an element of $\mathbf{1}^{\perp}$ the element of $\partial Y_{j}(e)$ reached ${ }^{6}$ by moving along the direction given by 1 . Hence, one can define a mapping $\Lambda_{j}: \mathbb{R}^{E} \times \mathbf{1}^{\perp} \rightarrow \bigcup_{e \in \mathbb{R}^{E}} \partial Y_{j}(e)$. This mapping is continuous according to Lemma 3.1 in (Bonnisseau 1997). It allows us to endow each $\partial Y_{j}(e)$ with the manifold structure defined by $\mathbf{1}^{\perp}$.

We then define the set

$$
\begin{gathered}
U=\left\{\left(p,\left(s_{j}\right),\left(\omega_{i}\right), e\right) \in S_{++} \times\left(\mathbf{1}^{\perp}\right)^{n} \times \mathbb{R}^{L m} \times \operatorname{int}\left(K_{E}\right) \mid\right. \\
\left.p \cdot\left(\sum_{j=1}^{n} \Lambda_{j}\left(e, s_{j}\right)+\sum_{i=1}^{m} \omega_{i}\right)>0\right\} .
\end{gathered}
$$

This set is an open subset of $\mathcal{H} \times\left(\mathbf{1}^{\perp}\right)^{n} \times \mathbb{R}^{L m} \times \mathbb{R}^{E}$ and hence an orientable manifold ${ }^{7}$. It will serve as a domain for the equilibrium correspondence. The choice of $U$ as domain is motivated by the fact that behavior of consumers can be meaningfully defined only for a non-negative wealth (what imposes the

\footnotetext{
6 there exists such an element thanks to the free-disposal assumption.

7 In the following, we consider the canonical orientation such that $\operatorname{deg}(I d, U)=1$, see appendix for further details on the degree.
} 
restriction of the domain to $U$ ), while the mathematical analysis requires a sufficiently structured set to elaborate upon, in our framework an orientable manifold (what imposes the extension of the domain to $U$ ).

\subsection{Survival and Revenue assumptions}

As is standard in the general equilibrium literature with increasing returns (see (Bonnisseau 1997), (Bonnisseau and Médecin 2001), (Jouini 1992a) and (Jouini 1992b)), survival assumptions will play a crucial role in our analysis. From the economic point of view, survival assumptions ensure that the economy can produce a positive wealth in a sufficiently large range of situations. From the mathematical point of view, they allow together with the property (3) of the demand that a tangential condition holds for the equilibrium correspondence.

Remark 2 The simplest form of survival assumption is the interiority of initial endowments in a pure exchange economy. In presence of increasing returns, the survival assumption must encompass the possibility of losses in the production sector and hence is of the form, for every $\left(p,\left(y_{j}\right), \omega^{\prime}\right) \in W$, $p \cdot\left(\sum_{j=1}^{n} y_{j}+\omega^{\prime}\right)>0$, where $p$ stands for the market price, $y_{j}$ the production of firm $j$ and $\omega^{\prime}$ a vector of initial ressources for the economy. The restriction the assumption imposes on the primitives of the economy may be measured by the size of the set $W$ on which one requires it to hold.

We will base our arguments on the following version of the survival assumption, which ensures the viability of the economic process for a given environment:

Assumption $(S A(e, \omega))$ For all $\left(p,\left(y_{j}\right)\right) \in S \times \prod_{j=1}^{n} Y_{j}(e)$ such that $p \in \bigcap_{j} \phi_{j}\left(y_{j}, e\right)$ and $\sum_{j=1}^{n} y_{j}+\sum_{i=1}^{m} \omega_{i} \geq 0$, one has

$$
p \cdot\left(\sum_{j=1}^{n} y_{j}+\sum_{i=1}^{m} \omega_{i}\right)>0 .
$$

This assumption simply consists in a parametrization by the environment of the standard version of the survival assumption (see e.g (Jouini 1992a)). In the following we shall first assume that for a reference (the initial) environment, assumption $S A\left(e_{0}, \omega^{\prime}\right)$ holds for every $\omega^{\prime} \geq \omega$, this will ensure the existence of an equilibrium for the fixed environment $e_{0}$. We will then show that the satisfaction of assumption $S A(e, \omega)$ for every environment $e \in K_{E}$ suffice to extend the existence result to equilibrium with external effects.

An alternative used in (Bonnisseau and Médecin 2001) to prove directly the existence of an equilibrium with external effects is to posit that the survival as- 
sumption holds for the environment actually determined by the agents' choices and for every $\omega^{\prime} \geq \omega$. Our condition are no weaker than theirs nor is the converse true. However our approach allows to expressly compare the sufficient conditions for the existence of equilibrium with and without external effects, more precisely to underline which additional conditions are needed to ensure the existence of equilibrium with external effects, when the existence of an equilibrium with fixed environment is guaranteed. This approach seems to us appropriate as one aims at modeling the influence on the economic equilibrium of the emergence of environmental feedbacks. Moreover from an empirical point of view, considering for example an observer or a decision maker who has to forecast the economic evolution for various climate change scenarios, it seems much easier to check that, for a certain range of environmental conditions, the survival assumption holds than to compute the exact correlation between the economic activity and the environment and then check that the condition holds.

We finally introduce a revenue assumption at the individual level in order to ensure that the working of the economy provides a positive wealth to every agent:

Assumption $(R(e, \omega))$ For all $\left(p,\left(x_{i}\right),\left(y_{j}\right)\right) \in S \times \prod_{i=1}^{m}\left(\mathbb{R}_{+}^{L}\right) \times \prod_{j=1}^{n} Y_{j}(e)$ such that $p \in \bigcap_{j} \phi_{j}\left(y_{j}, e\right) \cap S_{++}$and $\sum_{j=1}^{n} y_{j}+\sum_{i=1}^{m} \omega_{i} \geq 0$, one has

$$
p \cdot \omega_{i}+r_{i}\left(p,\left(y_{j}\right)\right)>0 .
$$

The range of the assumptions $R(e, \omega)$ covers any potential equilibrium but not the whole of $U$. In order to be able to define consumer's behavior inside this gap, we borrow the idea of Lemma 2 in (Jouini 1992a), and define on $U^{\prime}=\left\{\left(p,\left(y_{j}\right),\left(\omega_{i}\right)\right) \in S \times \prod_{j=1}^{n} \bigcup_{e \in \mathbb{R}^{E}} \partial Y_{j}(e) \times\left(\mathbb{R}_{+}^{L}\right)^{m} \mid p \cdot\left(\sum_{j=1}^{n} y_{j}+\sum_{i=1}^{m} \omega_{i}\right)>0\right\}$ the following auxiliary income functions:

Lemma 2 There exist continuous mappings

$$
\tilde{r}_{i}: U^{\prime} \rightarrow \mathbb{R}
$$

such that:

(1) For $\left(p,\left(y_{j}\right),\left(\omega_{i}\right)\right) \in U^{\prime}$, one has $\tilde{r}_{i}\left(p,\left(y_{j}\right),\left(\omega_{i}\right)\right)+p \cdot \omega_{i}>0$ for all $i$;

(2) For $\left(p,\left(y_{j}\right),\left(\omega_{i}\right)\right) \in U^{\prime}$ such that for all $i p \cdot \omega_{i}+r_{i}\left(p,\left(y_{j}\right)\right)>0$, one has $r_{i}\left(p,\left(y_{j}\right)\right)=\tilde{r}_{i}\left(p,\left(y_{j}\right),\left(\omega_{i}\right)\right)$ for all $i$;

(3) For $\left(p,\left(y_{j}\right),\left(\omega_{i}\right)\right) \in U^{\prime}$, one has $\sum_{i=1}^{m} \tilde{r}_{i}\left(p,\left(y_{j}\right),\left(\omega_{i}\right)\right)=p \cdot \sum_{j=1}^{n} y_{j}$.

Proof: It suffices to set following (Jouini 1992a), for $\left(p,\left(y_{j}\right),\left(\omega_{i}\right)\right) \in U^{\prime}$,

$$
\tilde{r}_{i}\left(p,\left(y_{j}\right),\left(\omega_{i}\right)\right):=(1-\theta(\rho)) \frac{\sum_{i=1}^{m} \rho_{i}}{m}+\theta(\rho) \rho_{i}-p \cdot \omega_{i}
$$


where,

- $\rho=\left(\rho_{i}\right)=p \cdot \omega_{i}+r_{i}\left(p,\left(y_{j}\right)\right)$

- $\theta(\rho)=\left\{\begin{array}{cc}1, \quad \text { if for all } i & \rho_{i}>0 \\ \frac{\sum_{i=1}^{m} \rho_{i}}{\sum_{i=1}^{m} \rho_{i}-m \inf _{k} \rho_{k}}, & \text { otherwise }\end{array}\right.$

In the following we will summarize consumer $i$ behavior by the demand with auxiliary income which we shall denote, for sake of simplicity, by $D_{i}\left(e, p,\left(s_{j}\right),\left(\omega_{i}\right)\right)$ instead of $D_{i}\left(e, p, \tilde{r}_{i}\left(p, \Lambda_{j}\left(e, s_{j}\right),\left(\omega_{i}\right)\right)+p \cdot \omega_{i}\right)$. This mapping is well-defined and upper semi-continuous with compact and convex values on $U$.

\subsection{Equilibrium Correspondence}

In order to study their relationships we have to encompass the equilibria with a fixed environment and the equilibria with external effects in related algebraic structures. Therefore, given an environment $e_{0} \in K_{E}$ we define the family of correspondences:

$$
F_{t}^{e_{0}}: U \rightarrow \mathcal{H} \times\left(\mathbf{1}^{\perp}\right)^{n} \times \mathbb{R}^{L m} \times \mathbb{R}^{E}
$$

by $F_{t}^{e_{0}}\left(p,\left(s_{j}\right),\left(\omega_{i}\right), e\right)=$

$$
\left(\begin{array}{c}
\left.\operatorname{proj}_{\mathcal{H}}\left(\sum_{i=1}^{m} D_{i}\left(e, p,\left(s_{j}\right),\left(\omega_{i}\right)\right)-\sum_{j=1}^{n} \Lambda_{j}\left(e, s_{j}\right)\right)-\sum_{i=1}^{m} \omega_{i}\right), \\
\left(\phi_{j}\left(e, \Lambda_{j}\left(e, s_{j}\right)\right)-p\right), \\
\left(w_{i}\right), \\
\left.e-\operatorname{proj}_{K_{E}}\left[t \xi\left(D_{i}\left(e, p,\left(s_{j}\right),\left(\omega_{i}\right)\right), \Lambda_{j}\left(e, s_{j}\right)\right)+(1-t) e_{0}\right)\right]
\end{array}\right)
$$

Those correspondences are upper semi-continuous, have compact and convex values, compact (pointwise) inverse image, and their domain and codomain are orientable manifolds of the same dimension. Hence they fit into the framework of Cellina's degree theory presented in the appendix. Moreover, they characterize the equilibria of $\mathcal{E}(\omega)$ in the sense of the following propositions:

Proposition 1 Assume assumption $R\left(e_{0}, \omega\right)$ holds.

One has $\left(p,\left(s_{j}\right), \omega, e\right) \in\left(F_{0}^{e_{0}}\right)^{-1}(\mathbf{1}, 0, \omega, 0)$ if and only if there exists $x_{i} \in D_{i}\left(e, p,\left(s_{j}\right),\left(\omega_{i}\right)\right)$ such that $\left.\left(p,\left(x_{i}\right),\left(\Lambda_{j}\left(e, s_{j}\right)\right)\right)\right)$ is an equilibrium of $\mathcal{E}(\omega)$ for the fixed environment $e_{0}$. 
Proposition 2 Assume assumption $R(e, \omega)$ holds for all $e \in K_{E}$.

One has $\left(p,\left(s_{j}\right), \omega, e\right) \in\left(F_{1}^{e_{0}}\right)^{-1}(\mathbf{1}, 0, \omega, 0)$ if and only if there exists $x_{i} \in D_{i}\left(e, p,\left(s_{j}\right),\left(\omega_{i}\right)\right)$ such that $\left.\left(p,\left(x_{i}\right),\left(\Lambda_{j}\left(e, s_{j}\right)\right)\right)\right)$ is an equilibrium with external effects of $\mathcal{E}(\omega)$.

Proposition 3 Assume assumption $R(e, \omega)$ holds for all $e \in K_{E}$.

One has $\left(p,\left(s_{j}\right), \omega, e\right) \in F_{t}^{-1}(e, 0, \omega, 0,0)$ if and only if there exists an environment $e^{\prime} \in K_{E}$ and $x_{i} \in D_{i}\left(e, p,\left(s_{j}\right),\left(\omega_{i}\right)\right)$ such that $\left.\left(p,\left(x_{i}\right),\left(\Lambda_{j}\left(e, s_{j}\right)\right)\right)\right)$ is an equilibrium of $\mathcal{E}(\omega)$ for the fixed environment $e^{\prime}$.

\section{Proof: (of proposition 1)}

Let $\left(p,\left(s_{j}\right), \omega, e\right) \in\left(F_{0}^{e_{0}}\right)^{-1}(\mathbf{1}, 0, \omega, 0)$. First of all, it is clear as $e_{0} \in K_{E}$ that $e=e_{0}$. Let us then set $\left.y_{j}=\Lambda_{j}\left(e_{0}, s_{j}\right)\right)$ and choose an element $x_{i} \in$ $D_{i}\left(e_{0}, p,\left(s_{j}\right),\left(\omega_{i}\right)\right)$, such that

$$
\operatorname{proj}_{\mathcal{H}}\left(x_{i}-\sum_{j=1}^{n} y_{j}-\sum_{i=1}^{m} \omega_{i}\right)=\mathbf{1} .
$$

Using Walras law (property 2 of Lemma 1), one then has $\sum_{i=1}^{m} x_{i}-\sum_{j=1}^{n} y_{j}=$ $\omega \geq 0$.

Hence, the $x_{i}$ are attainable for the environment $e_{0} \in K_{E}$ and lie in a compact set, what implies using property 3 of lemma 1 that $p \in S_{++}$. Moreover, it is clear that one has for all $j, p \in \phi_{j}\left(e_{0}, y_{j}\right)$. One can then use assumption $R\left(e_{0}, \omega\right)$, in order to ensure that the auxiliary incomes coincide with the original ones.

This suffices to prove that $\left(p,\left(x_{i}\right),\left(y_{j}\right)\right)$ is an equilibrium of $\mathcal{E}(\omega)$ for the fixed environment $e_{0}$.

The converse is straightforward.

\section{Proof: (of proposition 2)}

Let $\left(p,\left(s_{j}\right), \omega, e\right) \in\left(F_{0}^{e_{0}}\right)^{-1}(\mathbf{1}, 0, \omega, 0)$. Let us set $\left.y_{j}=\Lambda_{j}\left(e, s_{j}\right)\right)$ and choose an element $x_{i} \in D_{i}\left(e, p,\left(s_{j}\right),\left(\omega_{i}\right)\right)$, such that

$$
\operatorname{proj}_{\mathcal{H}}\left(x_{i}-\sum_{j=1}^{n} y_{j}-\sum_{i=1}^{m} \omega_{i}\right)=\mathbf{1}
$$

and

$$
e-\operatorname{proj}_{K_{E}} \xi\left(\left(x_{i}\right)\left(y_{j}\right)\right)=0 \text {. }
$$

Using Walras law, it is clear that $\sum_{i=1}^{m} x_{i}-\sum_{j=1}^{n} y_{j}=\omega \geq 0$, and hence that $\left(\left(x_{i}\right),\left(y_{j}\right)\right)$ is an attainable allocation for the environment $e$. It follows that 
$\xi\left(\left(x_{i}\right),\left(y_{j}\right)\right) \in K_{E}$ and therefore $e=\xi\left(\left(x_{i}\right),\left(y_{j}\right)\right)$ : the compatibility constraints between the economic activity and the environment are satisfied.

The remaining of the proof proceeds as in the case of proposition 1 except that one uses the fact that $e \in K_{E}$ and that $R\left(e^{\prime}, \omega\right)$ holds true for every $e^{\prime}$ in $K_{E}$ in order to show that the auxiliary incomes coincide with the original ones.

The proof of proposition 3 follows from the same arguments.

\section{Index Formula}

\subsection{Equilibria for a fixed environment}

We first establish an index formula for equilibria with a fixed environment $e_{0} \in \operatorname{int}\left(K_{E}\right)$. This fixed environment and the corresponding equilibria, whose existence is implied by the index formula, should be thought of as the situation which prevails before major environmental feedbacks have appeared. Taking as example the emergence of climate change impacts, the reference environment corresponds to the current (or recent past) situation where the effects on the temperature of the intensive use of fossil fuels are not significant from an economic point of view.

From the mathematical point of view, as mentioned above, the corresponding concept of equilibrium with fixed environment simply is a parametrized version of the standard one. It is therefore not surprising that the index formula established in (Jouini 1992a) can easily be generalized to this framework.

Theorem 1 Assume assumptions $(P),(P R),(C), R\left(e_{0}, \omega\right)$ and for all $\omega^{\prime} \geq \omega, S A\left(e_{0}, \omega^{\prime}\right)$, hold. The degree of $F_{0}^{e_{0}}$ at $(e, 0, \omega, 0,0)$ is equal to $(-1)^{L-1}$.

Corollary 1 There exist equilibria for a fixed environment in the economy $\mathcal{E}(\omega)$.

Proof: (of the corollary) This is a straightforward consequence of the property of non-triviality of the degree (see appendix).

Proof: (of the theorem) Using Theorem 5.1 in (Jouini 1992a) and its extensions in (Jouini 1992b) (see also (Bonnisseau 1992)), it is straightforward that in the cases where for all $j$, the pricing rules $\phi_{j}\left(e_{0},.\right)$ have bounded losses or coincide with the marginal pricing rule, the correspondence

$$
G^{e_{0}}: V \rightarrow \mathcal{H} \times\left(\mathbf{1}^{\perp}\right)^{n} \times \mathbb{R}^{L m}
$$


defined on

$$
\begin{gathered}
V:=\left\{\left(p,\left(s_{j}\right),\left(\omega_{i}\right)\right) \in S_{++} \times\left(\mathbf{1}^{\perp}\right)^{n} \times \mathbb{R}^{L m} \mid p \cdot\left(\sum_{j=1}^{n} \Lambda_{j}\left(e_{0}, s_{j}\right)+\sum_{i=1}^{m} \omega_{i}\right)>0\right\} \\
G^{e_{0}}\left(p,\left(s_{j}\right),\left(\omega_{i}\right)\right)=\left(\begin{array}{c}
\operatorname{proj}_{\mathcal{H}}\left(\sum_{i=1}^{m} D_{i}\left(e_{0}, p,\left(s_{j}\right),\left(\omega_{i}\right)\right)-\sum_{j=1}^{n} \Lambda_{j}\left(e_{0}, s_{j}\right)-\sum_{i=1}^{m} \omega_{i}\right), \\
\left(\phi_{j}\left(e_{0}, \Lambda_{j}\left(e_{0}, s_{j}\right)\right)-p\right), \\
\left(w_{i}\right) .
\end{array}\right)
\end{gathered}
$$

has degree $(-1)^{L-1}$.

Now, the family of correspondences $\left(H_{t}\right)_{t \in[0,1]}$ defined on $V \times \operatorname{int}\left(K_{E}^{m+n}\right)$ by

$$
H_{t}: t \rightarrow t\left(\left(G^{e_{0}}, 0\right)+(1-t) F_{0}^{e_{0}}\right.
$$

is, using the invariance by homotopy property of the degree, preserving the degree in $(\mathbf{1}, 0, \omega, 0)$ as all its relevant zeroes are in fact equilibria for the fixed environment $e_{0}$ and hence all lie in a compact set of $V \times \operatorname{int}\left(K_{E}^{m+n}\right)$. This ends the proof.

\subsection{Equilibria with external effects}

We now focus on the situation where feedbacks between the economic activity and the environment emerge ${ }^{8}$. Starting from the existence of equilibria for the reference environment $e_{0} \in K_{E}$, we can infer existence of equilibria with external effects, and even show that the degree of the equilibrium correspondence is preserved, provided that the survival assumption is satisfied along the path defined by the homotopy $F_{t}$, that is provided the economy is able to maintain a positive wealth creation as the feedbacks from the environment emerge.

Namely, we have:

Theorem 2 Assume the necessary conditions of theorem 1 hold and that moreover $S A(e, \omega)$ hold for all $e \in K_{E}$. One then has for all $t \in[0,1]$ :

$$
\operatorname{deg}\left(F_{t}^{e_{0}},(\mathbf{1}, 0, \omega, 0)\right)=(-1)^{L-1}
$$

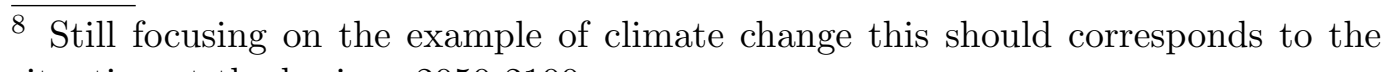
situation at the horizon 2050-2100. 
Proof: For sake of simplicity, we denote $F_{t}^{E_{0}}$ by $F_{t}$ in the course of the proof. We then show that the degree is preserved between $F_{0}$ and $F_{1}$. To actually prove invariance by homotopy of the degree, it suffices to show that $\cup_{\tau \in[0,1]} F_{t}^{-1}(e, 0, \omega, 0,0)$ is compact in $U$ (see appendix for further details).

Let us then consider a sequence $\left(p^{n},\left(s_{j}^{n}\right), \omega, e_{n}\right) \in \bigcup_{\tau \in[0,1]} F_{\tau}^{-1}(e, 0, \omega, 0,0)$ and show it has a converging subsequence with limit in $U$.

For all $n$ there exist $t^{n}$ such that $(\mathbf{1}, 0, \omega, 0) \in F_{t^{n}}\left(p^{n},\left(s_{j}^{n}\right), \omega, e_{n}\right)$. Let us then set $\left.y_{j}^{n}=\Lambda_{j}\left(e^{n}, s_{j}^{n}\right)\right)$ and choose an element $x_{i}^{n} \in D_{i}\left(e^{n}, p^{n},\left(s_{j}^{n}\right), \omega\right)$, such that

$$
\operatorname{proj}_{\mathcal{H}}\left(x_{i}^{n}-\sum_{j=1}^{n} y_{j}^{n}-\sum_{i=1}^{m} \omega_{i}\right)=\mathbf{1}
$$

and

$$
e^{n}-\operatorname{proj}_{K_{E}}\left(t_{n} \xi\left(\left(x_{i}^{n}\right),\left(y_{j}^{n}\right)\right)+\left(1-t_{n}\right) e_{0}\right)=0 .
$$

Using Walras law, one has $\sum_{i=1}^{m} x_{i}^{n}-\sum_{j=1}^{n} y_{j}^{n}=\omega \geq 0$.

This implies $\left(\left(x_{i}^{n}\right),\left(y_{j}^{n}\right)\right)$ is an attainable allocation for the environment $e_{n}$ and hence belongs to the compact set $K^{m+n}$.

Due to the continuity of the projection on $\mathbf{1}^{\perp}$ and the compacity of $K^{m+n}$, this implies that for all $j, s_{j}^{n}$ lie in a compact set.

Also, it is clear that $e^{n} \in K_{E}$.

Finally as $\phi_{j}$ has values in $S$, one has $p^{n} \in S$.

To sum up, $\left(p^{n},\left(s_{j}^{n}\right), \omega, e^{n},\left(x_{i}^{n}\right),\left(y_{j}^{n}\right) t^{n}\right)$ belongs to the compact set $S \times\left(\mathbf{1}^{\perp}\right)^{n} \times$ $\mathbb{R}^{L m} \times K_{E} \times K^{m+n} \times[0,1]$ and hence has a subsequence converging inside this set. Let us denote by $\left(p,\left(s_{j}\right),\left(\omega_{i}\right), e,\left(x_{i}\right),\left(y_{j}\right), t\right)$ its limit. It remains to show that $\left(p,\left(s_{j}\right),\left(\omega_{i}\right), e\right)$ is in $U$.

It is clear by continuity that $y_{j}=\Lambda_{j}\left(e, s_{j}\right), x_{i} \in D_{i}\left(e, p,\left(s_{j}\right), \omega\right)$ and that $\sum_{i=1}^{m} x_{i}-\sum_{j=1}^{n} y_{j}=\omega \geq 0$.

This first implies that $\left(\left(x_{i}\right),\left(y_{j}\right)\right) \in K^{m+n}$, and hence that $\xi\left(\left(x_{i}\right),\left(y_{j}\right)\right)$ belongs to the interior of $K_{E}$. It is also the case that $e_{0} \in \operatorname{int}\left(K_{E}\right)$ by assumption. Now, one has by continuity that $e=\operatorname{proj}_{K_{E}}\left(t \xi\left(\left(x_{i}\right),\left(y_{j}\right)\right)+(1-t) e_{0}\right)$, so that the preceding implies $e \in \operatorname{int}\left(K_{E}\right)$.

Second, continuity properties of $\phi_{j}$ imply that $p \in \phi_{j}\left(e, y_{j}\right)$. As moreover $\sum_{j=1}^{n} y_{j}+\sum_{i=1}^{m} \omega_{i} \geq 0$ and e belongs to the interior of $K_{E}$, the survival assumption $S A(e, \omega)$ implies that $p \cdot\left(y_{j}+\sum_{i=1}^{m} w_{i}\right)>0$ and therefore $\tilde{r}_{i}\left(p, \Lambda_{j}\left(E_{t}, s_{j}\right), \omega_{i}\right)+$ $p \cdot \omega_{i}>0$. Given the fact that $\left(x_{i}\right)$ is bounded, the boundary condition (3) in Lemma 1 then implies that $p \in S_{++}$. This ends the proof. 
Using the property of non-triviality of the degree, Theorem 2 provides a serie of existence results for equilibria with external effects :

Corollary 2 Under the assumptions ${ }^{9}$ of Theorem 2, there exists an equilibrium in the economy. $\mathcal{E}(\omega)$.

In particular, one has for loss free pricing rules for which the survival assumptions are satisfied as soon as the initial endowments satisfy an interiority condition:

Corollary 3 Under assumptions $(P),(P R),(C)$, if for all $i \omega_{i} \in \mathbb{R}_{++}^{L}$ and if the pricing rules are loss-free, there exists an equilibrium with external effects in the economy $\mathcal{E}(\omega)$.

This encompasses the case of competitive behavior:

Corollary 4 Under assumptions $(P)$ and $(C)$, if for all $i\left(\omega_{i}\right) \in \mathbb{R}_{++}^{L}$ and if for all $j$ the production correspondences have convex values containing 0 while the producers maximize their profit, there exists an equilibrium with external effects in the economy $\mathcal{E}(\omega)$.

Proof: Indeed, in this framework, the pricing rule coincide with the restriction to $S$ of the normal cone of convex analysis and satisfy all the properties required by theorem 2, (in particular assumption $P R$ )

Finally, one should note that from the non-nullity of the degree of the correspondences $F_{t}$ for $\left.t \in\right] 0,1[$ one can infer the existence of equilibria of $\mathcal{E}(\omega)$ for undetermined environment in $K_{E}$ corresponding to situations where the environmental effects are only partially taken into account. Even-tough those equilibria are not of a tremendous interest per se, their existence can be seen as indicating the possibility of a transition between an equilibrium with a fixed environment and an equilibrium with external effects through an equilibrium path. Such transitions will be further studied in the following section.

\footnotetext{
9 One should have in mind that according to (Bonnisseau and Médecin 2001) in the case of marginal pricing, Clarke's normal cone does not necessarily satisfy the first condition of assumption (PR) because its graph may not be closed.Sufficient conditions for the marginal pricing rule to satisfy the assumption (PR) is that $Y_{j}$ has convex values or that an additional smoothness requirement hold (see assumption (PS) in (Bonnisseau and Médecin 2001)).
} 


\section{Regular Economies and the Equilibrium Manifold}

In order to fully exploit the preceding results : use the index formula to actually count the number of equilibria or study the sensitivity of the equilibria to environmental feedbacks, we need to gain further insight on the structure of the set of equilibria.

Therefore, we aim at extending the results of (Jouini 1992C) to our framework. We shall in the following assume that the necessary assumptions of Theorem 2 hold and moreover that $\xi,\left(\phi_{j}\right)_{j=1 \cdots n},\left(D_{i}\right)_{i=1 \cdots}$ and $\left(r_{i}\right)_{i=1 \cdots m}$ are $C^{k}$ functions for some $k \geq 1$.

Additionally, we introduce a first transversality condition on the pricing rules which is the equivalent of assumption TR of (Jouini 1992C):

Assumption (TR1) For every $\left(e,\left(y_{j}\right), p\right) \in K_{E} \times \prod_{j=1}^{n} Y_{j} \times S_{++}$such that $p \in \bigcap_{j} \phi_{j}\left(e, y_{j}\right)$, the matrix

$$
\left(\begin{array}{ccccc}
\frac{\partial \phi_{1}\left(e, y_{1}\right)}{\partial y_{1}} & 0 & \ldots & 0 & -I_{L-1} \\
0 & \ddots & \ddots & \vdots & \vdots \\
\vdots & \ddots & \ddots & 0 & \vdots \\
0 & \ldots & 0 & \frac{\partial \phi_{n}\left(e, y_{n}\right)}{\partial y_{n}} & -I_{L-1}
\end{array}\right)
$$

has rank $n(L-1)$

This assumption ensures that the pricing rules do not have "too many " common critical values and amounts to assume that $0 \in \mathcal{H}^{n}$ is a regular value of the mapping defined on $\mathcal{H} \times\left(\mathbf{1}^{\perp}\right)^{n} \times \mathbb{R}^{E}$ and with values in $\mathcal{H}^{n}$ :

$$
\left(p,\left(y_{j}\right), e\right) \rightarrow\left(\phi_{j}\left(e, \Lambda_{j}\left(e, s_{j}\right)\right)-p\right)_{j=1 \cdots n}
$$

According to Sard's Lemma, almost every value of this mapping is regular, so that assumption TR1 is always satisfied modulo an $\epsilon$-perturbation of the pricing rules (see (Jouini 1992C)).

Under this assumption, the set of equilibria with an environment in $\operatorname{int}\left(K_{E}\right)$ has a manifold structure:

Proposition 4 Under assumptions $T R$, the set of equilibria with a fixed environment in int $\left(K_{E}\right)$ is a $C^{k}$ submanifold of $\mathcal{H} \times\left(\mathbf{1}^{\perp}\right)^{n} \times \mathbb{R}^{L m} \times \mathbb{R}^{E}$ with dimension $m L+E$. 
Proof: It is a straightforward extension of proposition 3.1 in (Jouini 1992C) that under assumption (TR1) the $C^{k}$ mapping $H$ with values in $\mathcal{H} \times\left(\mathbf{1}^{\perp}\right)^{n}$ and defined on $U$ by $H\left(p,\left(s_{j}\right),\left(\omega_{i}\right), e\right)=$

$$
\left.\begin{array}{c}
\operatorname{proj}_{\mathcal{H}}\left(\sum_{i=1}^{m} D_{i}\left(e, p, \tilde{r}_{i}\left(p, \Lambda_{j}\left(e, s_{j}\right),\left(\omega_{i}\right)\right)+p \cdot \omega_{i}\right)-\sum_{j=1}^{n} \Lambda_{j}\left(e, s_{j}\right)-\sum_{i=1}^{m} \omega_{i}\right), \\
\left(\phi_{j}\left(e, \Lambda_{j}\left(e, s_{j}\right)\right)-p\right) .
\end{array}\right)
$$

is a submersion in $(0,0)$.

Hence $G^{-1}(0,0)$, which is exactly the set of equilibria for a fixed environment in int $K_{E}$ is a submanifold of dimension $m L+E$ of $\mathcal{H} \times\left(\mathbf{1}^{\perp}\right)^{n} \times \mathbb{R}^{L m} \times \mathbb{R}^{E}$ (as this set is non-empty according to the results of the preceding section).

The remaining results of (Jouini 1992C), in particular proposition 4.4, could then easily be extended to our framework, in order to show that in the neighborhood of almost every $(\omega, e)$, there is a finite parametrization of the equilibrium set by $C^{k}$ functions depending on the initial endowment and the environment. Therefore, there is a strong confidence that the economy can react to a change of its environment without too brusque transitions before it reaches, after the feedbacks between the economic activity and the environment have been completly established, an equilibria with external effects.

There may however be a large indeterminacy on the equilibrium with external effects reached ; for example up to now nothing prevents the existence of an infinite number of equilibria with external effects.

Part of this indeterminacy can be lifted using the index formula established in Theorem 2. Indeed, let us call regular the economy $\mathcal{E}(\omega)$ whenever $(\mathbf{1}, 0, \omega, 0)$ is a regular value of $F_{1}^{e_{0}}{ }^{10}$. It is then a direct application of the degree theory of $C^{k}$ functions on finite dimensional open sets that for a regular economy $\mathcal{E}(\omega)$

$$
\operatorname{deg}\left(F_{1}^{e_{0}},(\mathbf{1}, 0, \omega, 0)\right)=\sum_{\left(p,\left(s_{j}\right), \omega, e\right) \in\left(F_{1}^{e_{0}}\right)^{-1}(\mathbf{1}, 0, \omega, 0)} I_{\left(p,\left(s_{j}\right), \omega, e\right)}
$$

where $I_{\left(p,\left(s_{j}\right), \omega, e\right)}$ the index of the "equilibrium" $\left(p,\left(s_{j}\right), \omega, e\right)$ is equal to the sign of the determinant of the differential of $F_{1}^{e_{0}}$ at $\left(p,\left(s_{j}\right), \omega, e\right)$. This immediately implies, using Theorem 2, that:

Proposition 5 If $\mathcal{E}(\omega)$ is regular, than it has an odd number of equilibria. Moreover, if the index is constant over the set of equilibria, $\mathcal{E}(\omega)$ has an unique equilibrium.

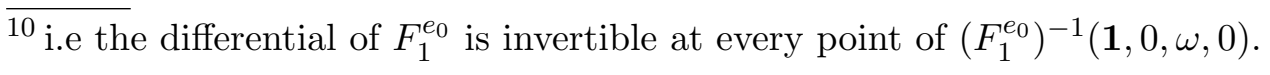


This result is however useful only if one can ensure that a large number of economies are regular. This is what we now aim at proving.

Therefore, one requires that a second transversality condition holds :

Assumption (TR2) For every $\left(e,\left(y_{j}\right), p\right) \in K_{E} \times \prod_{j=1}^{n} Y_{j} \times S_{++}$such that there exists $\omega \in\left(\mathbb{R}_{+}^{L}\right)^{m}$ with $H\left(p,\left(s_{j}\right), \omega, e\right)=0^{11}$ the matrix

$$
I_{E}-\left(\frac{\partial \xi}{\partial x_{1}}, \cdots, \frac{\partial \xi}{\partial x_{m}}, \frac{\partial \xi}{\partial y_{1}}, \cdots, \frac{\partial \xi}{\partial y_{n}}\right)\left(\frac{\partial D_{1}}{\partial e}, \cdots, \frac{\partial D_{m}}{\partial e}, \frac{\partial \Lambda_{1}}{\partial e}, \cdots, \frac{\partial \Lambda_{n}}{\partial e}\right)^{T}
$$

has full rank.

This condition on the differential of $e \rightarrow e-\xi\left(D_{i}\left(e, p,\left(s_{j}\right),\left(\omega_{i}\right)\right), \Lambda_{j}\left(e, s_{j}\right)\right)$ is always satisfied modulo a small perturbation of the environmental function (a direct application of Sard's lemma as in (Jouini 1992C)). It states that a local change in the state of the environment does not provoke changes in the agent's consumption and production which would in turn entail the exact same changes in the state of the environment. Finally, it allows us to ensure that the set of equilibria with external effects also has a manifold structure:

Proposition 6 Under assumptions $T R 1$ and $T R 2$ the set of equilibria with external effects is a $C^{k}$ submanifold of dimension $m L$.

Proof: It is a direct extension of proposition 4 that under assumption (TR1) and TR2 the $C^{k}$ mapping $J$ with values in $\mathcal{H} \times\left(\mathbf{1}^{\perp}\right)^{n} \times \mathbb{R}^{E}$ and defined on $U$ by $J\left(p,\left(s_{j}\right),\left(\omega_{i}\right), e\right)=$

$$
\left(\begin{array}{c}
\operatorname{proj}_{\mathcal{H}}\left(\sum_{i=1}^{m} D_{i}\left(e, p, \tilde{r}_{i}\left(p, \Lambda_{j}\left(e, s_{j}\right),\left(\omega_{i}\right)\right)+p \cdot \omega_{i}\right)-\sum_{j=1}^{n} \Lambda_{j}\left(e, s_{j}\right)-\sum_{i=1}^{m} \omega_{i}\right), \\
\left(\phi_{j}\left(e, \Lambda_{j}\left(e, s_{j}\right)\right)-p\right) \\
e-\xi\left(D_{i}\left(e, p,\left(s_{j}\right),\left(\omega_{i}\right)\right), \Lambda_{j}\left(e, s_{j}\right)\right)
\end{array}\right)
$$

is a submersion in $(0,0,0)$.

Hence $J^{-1}(0,0)$, which is exactly the set of equilibria with external effects is a submanifold of dimension $m L$ of $\mathcal{H} \times\left(\mathbf{1}^{\perp}\right)^{n} \times \mathbb{R}^{L m} \times \mathbb{R}^{E}$ (as it is non-empty according to the results of the preceding section).

One can then apply Sard's lemma in order to show that the projection from the manifold of equilibrium with external effects onto the space of initial allocations $\left(\mathbb{R}_{+}^{L}\right)^{m}$ is almost everywhere regular, what implies that for almost every $\omega \in\left(\mathbb{R}_{+}^{L}\right)^{m}$, the economy $\mathcal{E}(\omega)$ is regular. Hence Proposition 5 generically applies.

$\left.\overline{{ }^{11} \text { i.e }(e},\left(y_{j}\right), p\right)$, entails an equilibrium for some $\omega$. 


\section{Appendix}

\subsection{Degree theory (mostly taken from (Jouini 1992a))}

The degree theory of upper-semi-continuous correspondences developped in (Granas 1959) and (Cellina and Lasota 1969) is an extension of the topological degree theory. Given an upper-semi-continuous correspondence $F$ with convex and compact values, there exists for every $\epsilon>0$, a continuous function $f_{\epsilon}$ whose graph is contained in an $\epsilon$-neighborhood of the graph of $F$. Approximating $f_{\epsilon}$ by a smooth function, one can define the degree of $F$ as the degree of those smooth approximations for $\epsilon$ sufficiently small ( see (Cellina and Lasota 1969), (Giraud 2001)). More precisely, one obtains the following axiomatic characterization.

Given the set $C$ of $(F, X, Y, y)$ where:

(1) $X$ and $Y$ are two oriented manifolds of the same dimension contained in an euclidian space;

(2) $F: X \rightarrow Y$ is an upper semi-continuous correspondence with nonempty convex compact values,

(3) $y \in Y$ and $F^{-1}(y):=\{x \in X \mid y \in F(x)\}$ is a compact subset of $X$.

Theorem 3 There exists a unique function, called degree, deg: $C \rightarrow \mathbb{Z}$ such that:

(1) (Normalization) $\operatorname{deg}(I d, Y, Y, y)=1$

(2) (Localization) If $F^{-1}(y) \subset U$ and $F(U) \subset V$ where $U$ and $V$ are open subsets of $X$ and $Y$ respectively, then:

$$
\operatorname{deg}(F, X, Y, y)=\operatorname{deg}\left(F_{\mid U}, U, V, y\right)
$$

(3) (Additivity) If $\left(G_{i}\right)_{i=1 \cdots n}$ is a finite partition of $X$ by open sets such that for all $i,\left(F_{\mid G_{i}}, G_{i}, Y, y\right) \in C$, then

$$
\operatorname{deg}(F, X, Y, y)=\sum_{i=1}^{n} \operatorname{deg}\left(F_{\mid G_{i}}, G_{i}, Y, y\right)
$$

(4) (Invariance by homotopy) If $\left(F_{t}, X, Y, y\right)$ is a family of elements of $C$ such that for every compact subset $K$ of $X$ the mapping $t \rightarrow \operatorname{Graph}_{t} \cap(K \times Y)$ is continuous with regard to the Hausdorff's distance on $K \times Y$ and such that $\bigcup_{t \in[0,1]} F_{t}^{-1}(y)$ is a compact subset of $X$, then:

$$
\operatorname{deg}\left(F_{0}, X, Y, y\right)=\operatorname{deg}\left(F_{1}, X, Y, y\right)
$$


(5) (Continuity)If there exists a compact neighborhood, $K$ of $y^{*}$ such that $F^{-1}(K):=\{x \in X \mid F(x) \cap K \neq \emptyset\}$ is compact, then $\operatorname{deg}(F, X, Y, y)$ is constant on $K$.

(6) (Chain Rule) If $(F, X, Y, y)$ and $(G, Y, Z, z)$ are two elements of $C$ such that $Y$ is connected and for every compact $K$ of $Y, F^{-1}(K)$ is compact, then

$$
\operatorname{deg}(G \circ F, X, Z, z)=\operatorname{deg}(F, X, Y, y) \cdot \operatorname{deg}(G, Y, Z, z)
$$

where for all $x G \circ F(x):=\operatorname{clc}\left(\bigcup_{y \in F(x)} G(y)\right)$

(7) (Non-triviality) If $\operatorname{deg}(F, X, Y, y) \neq 0$ then $F^{-1}(y) \neq 0$.

\subsection{Externalities and free-disposability}

To illustrate the point made in Remark 1, let us consider two firms operating on two goods. A production plan of the first firm being denoted by $\left(x_{1}, x_{2}\right) \in$ $\mathbb{R}^{2}$ and one of the second firm being denoted by $\left(y_{1}, y_{2}\right) \in \mathbb{R}^{2}$. Consider a situations where both producers are affected by the level of a certain pollutant $e \in \mathbb{R}$. For example, one can consider that production possibilities are given by the same production correspondence for the two firms :

$$
Y(e)=\left\{\left(z_{1}, z_{2}\right) \mid z_{2} \leq \min \left(-z_{1}, 1-e\right) \text { and } z_{1} \leq 0\right\}
$$

For such a representation of the production possibilities, which is the one used in the paper, the free-disposal assumption is satisfied independently of the correlations between the pollution and the production process.

Now, consider that the level of pollution is determined by the total use of good 1 in the economy. For example that when the production plans are $\left(x_{1}, x_{2}\right)$ and $\left(y_{1}, y_{2}\right)$ respectively, the total level of pollution is $-x_{1}-y_{1}$. In our framework this simply means that the environmental function is

$$
\xi:\left(\left(x_{1}, x_{2}\right),\left(y_{1}, y_{2}\right)\right) \rightarrow-x_{1}-y_{1}
$$

However, if one then wants to write the production correspondence in the framework of (Laffont 1978), one should write :

$$
\begin{aligned}
& Y_{1}\left(\left(y_{1}, y_{2}\right)\right)=\left\{\left(x_{1}, x_{2}\right) \mid x_{2} \leq \min \left(-x_{1}, 1+x_{1}+y_{1}\right) \text { and } x_{1} \leq 0\right\} \\
& Y_{2}\left(\left(x_{1}, x_{2}\right)\right)=\left\{\left(y_{1}, y_{2}\right) \mid y_{2} \leq \min \left(-y_{1}, 1+y_{1}+x_{1}\right) \text { and } y_{1} \leq 0\right\}
\end{aligned}
$$

In this setting ,the free-disposability assumption is no longer satisfied. 


\section{References}

Bonnisseau, J-M. (1997) "Existence of Equilibria in Economies with Externalities and Nonconvexities." Set-Valued Analysis, Volume 5, Number 3, pp. 209-226(18)

Bonnisseau, J-M. (2003) "Regular economies with non-ordered preferences." Special issue on the Athens-Minnesota Conferences . J. Math. Econom. 39, no. 3-4, 153-174.

Bonnisseau, J-M. and Cornet, B. (1988) "Existence of equilibria when firms follow bounded losses pricing rules." J. Math. Econom. 17 (1988), pp 193-207 Bonnisseau, J-M. and Médecin, J-P (2001). "Existence of marginal pricing equilibria in economies with externalities and non-convexities." J. Math. Econom. 36, no. 4, 271-294.

Bonnisseau, J-M. (1992) "Existence of equilibria in the presence of increasing returns : A synthesis" J. Math. Econom. vol. 21(5), pp 441-452.

Cellina, A. and Lasota, A. (1969). - "A New Approach to the Definition of Topological Degree for Multivalued Mappings", Atti della Academia Nazionale dei Lincei, Rendiconti. Classe de Scienze Fisiche, Mathematiche e Naturali, 47, pp. 434-440.

Clarke, F. (1983), "Optimization and nonsmooth analysis" Wiley, New York. Del Mercato, E. L.(2006), "Existence of competitive equilibria with externalities: A differential viewpoint" J. Math. Econom. 42 525-543.

Dierker, E. (1982). "Regular Economies" in Handbook of Mathematical Economics, Vol. 2, edited by K. J. Arrow and M. D. Intriligator, New York, North Holland.

Dierker, E. (1974). "Topological Methods in Walrasian Economics." Lecture notes on Economics and mathematical Sciences, 92. Springer-Verlag: Berlin. Florenzano, M. (2003) "General Equilibrium Analysis. Existence and Optimality of Equilibria" Kluwer, Boston/Dordrecht/London.

Giraud, G. (2001) "An algebraic index theorem for non-smooth economies" Journal of Mathematical Economics, V. 36( 4), pp. 255-269.

Granas, A. (1959), "Sur la notion de degré topologique pour une certaine classe de transformations multivalentes dans les espaces de Banach", Bull. Acad. Polon. Sci., Série Sc. Math. Astronom. Phys., 7 , 191-194.

Jouini, E. (1992) "An Index Theorem for Nonconvex Production Economies", Journal of Economic Theory, 57 (1), 176-196, 1992.

Jouini, E. (1992) "Unicité et stabilité de l'équilibre dans une économie de production avec règle de tarification marginale: les cas convexe et non-convexe", Annales d'Economie et de Statistique, 44: 159-176.

Jouini, E. (1992) "Structure de l'ensemble des équilibres d'une économie productive", Annales de l'Institut Henri Poincaré, Analyse non-linéaire, 9 (3), 321-336. 
Kehoe,T. (1980) "An index theorem for general equilibrium models with production", Econometrica. 48(5): 1211-32

Laffont, J, J. (1978) "Effets externes et théorie économique", Monographie du Séminaire d'économétrie. Centre National de la Recherche Scientifique, Paris.

Mas-Colell, A (1985) "The Theory of General Economic Equilibrium : A Differentiable Approach" Cambridge University Press, Cambridge, UK.

Starret, D.A. (1972) "Fundamental non convexities in the theory of externalities "Journal of Economic Theory n ${ }^{\circ} 4: 180-199$. 